\title{
The Uric Acid and Gout have No Direct Causality With Osteoarthritis: A Mendelian Randomization Study
}

\author{
Young Ho Lee, M.D., Ph.D., Gwan Gyu Song, M.D., Ph.D. \\ Department of Rheumatology, Korea University College of Medicine, Seoul, Korea
}

\begin{abstract}
Objective. To examine whether uric acid level or gout is causally associated with the risk of osteoarthritis. Methods. We performed a two-sample Mendelian randomization (MR) analysis using inverse-variance weighted (IVW), MR-Egger regression, and weighted median methods. We used the publicly available summary statistics datasets of uric acid level or gout genome-wide association studies (GWASs) as the exposure, and a GWAS in 3,498 patients with osteoarthritis in the arcOGEN study and 11,009 controls of European ancestry as the outcome. Results. Six single nucleotide polymorphisms (SNPs) from the GWAS data on uric acid level and 12 SNPs from the GWAS data on gout were selected as instrumental variables (IVs). The IVW analysis did not support a causal association between uric acid level or gout and risk of osteoarthritis (beta $=-0.026$, standard error $[\mathrm{SE}]=0.096, \mathrm{p}=0.789$; beta $=-0.018, \mathrm{SE}=0.025, \mathrm{p}=0.482$ ). MR-Egger regression revealed no causal association between uric acid level or gout and risk of osteoarthritis (beta $=0.028, \mathrm{SE}=0.142, p=0.852$; beta $=-0.056, \mathrm{SE}=0.090, p=0.548$ ). Similarly, no evidence of a casual association was provided by the weighted median approach (beta $=0.004, \mathrm{SE}=0.064$, $p=0.946$; beta $=-0.005, S E=0.025, p=0.843$ ). Conclusion. The results of MR analysis demonstrates that uric acid level and gout may be not causally associated with the increased risk of osteoarthritis. Considering MR study is not susceptible to bias from unmeasured confounders or reverse causation, the epidemiological evidence for an association between uric acid level or gout and a higher risk of osteoarthritis may be due to residual confounding or reverse causation rather than direct causality. (J Rheum Dis 2020;27:88-95)
\end{abstract}

Key Words. Uric acid, Gout, Osteoarthritis, Mendelian randomization analysis

\section{INTRODUCTION}

Osteoarthritis is the most common disease of joints, characterized by a progressive degeneration of articular cartilage, joint pain, and immobility [1]. It is a major cause of pain and disability in elderly people, and its prevalence continues to increase worldwide [2]. The health burden of osteoarthritis is growing with increase in the average longevity worldwide. Therefore, a comprehensive understanding of the risk factors associated with the occurrence of osteoarthritis is needed. Hyperuricemia is a condition in which the serum urate level exceeds the limit of urate solubility $(6.8 \mathrm{mg} / \mathrm{dL})$, which reflects supersaturation of the extracellular fluid with urate, and pre- disposes the body to gout [3]. Gout is an inflammatory disorder characterized by hyperuricemia and effects of urate crystal deposition, including episodic gout flares, gouty arthropathy, tophi, and urolithiasis [3]. Hyperuricemia leads to the deposition of monosodium urate (MSU) crystals in tissues, and increase in the levels of hyperuricemia is correlated with increasing incidences of gouty arthritis [4].

Epidemiological studies have demonstrated that higher serum uric acid levels and gout are associated with an increased risk of osteoarthritis, suggesting uric acid and gout as possible risk factors for osteoarthritis $[5,6]$. The relationship among hyperuricemia, gout, and osteoarthritis may be explained by the finding that MSU crystal

Received : August 20, 2019, Revised : October 24, 2019, Accepted : November 15, 2019

Corresponding to : Young Ho Lee iD http://orcid.org/0000-0003-4213-1909

Department of Rheumatology, Korea University Anam Hospital, Korea University College of Medicine, 73 Inchon-ro, Seongbuk-gu, Seoul 02841, Korea. E-mail : lyhcgh@korea.ac.kr

Copyright (c) 2020 by The Korean College of Rheumatology. All rights reserved.

This is an Open Access article, which permits unrestricted non-commerical use, distribution, and reproduction in any medium, provided the original work is properly cited. 
deposition on/in cartilage may create local mechanical and/or inflammatory damage, promoting osteoarthritis development, and that uric acid transporters are present on human articular chondrocytes, suggesting that these cells may internalize uric acid with potential pro-oxidant effects [7]. However, the shared risk factors due to obesity and aging represent a common feature among hyperuricemia, gout and osteoarthritis [8]. Hyperuricemia and gout may predispose joints to osteoarthritis, and osteoarthritis-caused cartilage damage may promote MSU deposition; further these conditions may trigger or result in shared inflammatory cascades. Observational studies are prone to biases, such as reverse causation interpretations and residual confounding, preventing a clear understanding of the effects of uric acid level and gout on osteoarthritis.

Mendelian randomization (MR) is a technique that uses genetic variants as instrumental variables (IVs) to assess whether an observational association between a risk factor and an outcome is consistent with a causal effect [9]. However, to date, MR has not been used to explore the causal effects of uric acid level and gout on the risk of osteoarthritis. The aim of this study was to examine, using a two-sample MR analysis, whether uric acid level and gout are causally associated with the risk of osteoarthritis.

\section{MATERIALS AND METHODS}

\section{Data sources and selection of genetic variants}

We searched NHGRI-EBI GWAS (https://www.ebi. ac.uk/gwas), a comprehensive catalog of reported associations from published GWAS studies. We used publicly available summary statistics datasets from uric acid level or gout GWASs. For the exposure dataset, we used publicly available summary statistics datasets from a metaanalysis of GWASs on uric acid levels from 14 studies with a total 28,141 participants of European descent [10], and three GWASs on gout (European: $\mathrm{n}=968$ cases and 15,506 controls [11]; Japanese: $\mathrm{n}=945$ cases and 1,213 controls, followed by 1,048 cases and 1,334 controls [12]), and replicated the results using a dataset from a GWAS with 1,396 cases and 1,268 controls from Caucasian and New Zealand Polynesian populations [13]. We used summary statistics from a GWAS based on 3,498 patients with osteoarthritis of the knee and hip in the arcOGEN study and 11,009 controls from the UK or European descent, as an outcome [14]. A two-sample MR study uses genetic variants associated with uric acid level or gout as
IVs to improve the inference. We obtained summary statistics (beta coefficients and standard errors [SE]) for single-nucleotide polymorphisms (SNPs) associated with uric acid level and gout as IVs from uric acid level and gout GWASs, and utilized the summary data of SNPs from osteoarthritis GWAS as an outcome. The 'top hits' from a GWAS can be used to define genetic instruments for exposures in MR analyses. The selection criteria for IVs were as follows; 1) Association p-values of SNPs with exposure should be less than a threshold of $\mathrm{p}<2.04 \mathrm{e}-05$ in GWAS dataset of exposure. 2) The SNPs from exposure dataset also should be present in GWAS dataset of outcome.

\section{Statistical analysis for Mendelian randomization} MR analysis requires genetic variants to be related to, but not potential confounders of, an exposure [15]. First, we assessed the independent association of SNPs with uric acid level or gout. Second, we examined the association between each SNP and the risk of osteoarthritis. Third, we combined these findings to estimate the uncompounded causal association between uric acid level or gout and osteoarthritis risk using MR analysis. We performed two-sample MR, which is a method used to estimate the causal effect of an exposure (uric acid level and gout) on outcomes (osteoarthritis) using summary statistics from different GWASs [16], to assess the causal relationships between uric acid level or gout and osteoarthritis risk.

The IVW method uses a meta-analysis approach to combine the Wald ratio estimates of the causal effect obtained from different SNPs, and provides a consistent estimate of the causal effect of the exposure on the outcome when each of the genetic variants satisfies the assumptions of an instrumental variable [17]. Although the inclusion of multiple variants in an MR analysis results in increased statistical power, it has the potential to include pleiotropic genetic variants that are not valid IVs [16]. To explore and adjust for pleiotropy (association of genetic variants with more than one variable), the weighted median and MR-Egger regression methods were used. MR-Egger regression analysis tests and accounts for the presence of unbalanced pleiotropy by introducing a parameter for this bias, through incorporation of summary data estimates of causal effects from multiple individual variants, making it robust to invalid instruments [18]. MR-Egger method performs a weighted linear regression of the gene-outcome coefficients on the gene-exposure coefficients [18]. The slope of this regression represents the causal effect 
estimate, and the intercept can be interpreted as an estimate of the average horizontal pleiotropic effect across the genetic variants [19]. The weighted median estimator provides a consistent estimate of the causal effect, even when up to $50 \%$ of the information contributing to the analysis comes from genetic variants that are invalid IVs [20]. The weighted median estimator has the advantage of retaining greater precision in the estimates than those from the MR-Egger analysis [20]. Tests were considered statistically significant at $\mathrm{p}<0.05$. All MR analyses were performed on MR Base platform [21].

\section{Heterogeneity and sensitivity test}

We assessed heterogeneities among SNPs using Cochran's Q-statistics and funnel plots [22]. We also performed a "leave one out" analysis to investigate the possibility that the causal association was driven by a single SNP.

\section{RESULTS}

\section{Studies included in the meta-analysis}

1) Instrumental variables for Mendelian randomization

We selected six SNPs as IVs from the GWAS data on uric acid level and 12 SNPs from the GWAS data on gout as IVs to improve the inference (Table 1, Figure 1). Two of the IVs (rs2231142, rs734553) were associated with both uric acid level and gout (Table 1). The selected IVs were associated with uric acid level and gout at genome-wide significance, except for rs10791821, 11733284, and rs734553 in gout (Table 1).

Table 1. Instrumental SNPs from uric acid level (A) or gout (B) and osteoarthritis GWASs

\begin{tabular}{|c|c|c|c|c|c|c|c|c|c|c|}
\hline \multicolumn{11}{|c|}{ A. Uric acid levels } \\
\hline \multirow{2}{*}{$\begin{array}{l}\text { Instrumental } \\
\text { SNP }\end{array}$} & \multirow{2}{*}{$\begin{array}{l}\text { Effect } \\
\text { allele }\end{array}$} & \multirow{2}{*}{ Gene } & \multicolumn{3}{|c|}{ Exposure (uric acid) } & \multicolumn{5}{|c|}{ Outcome (osteoarthritis) } \\
\hline & & & Beta & SE & $p$-value & Case (n) & Control (n) & Beta & SE & $\mathrm{p}$-value \\
\hline rs12356193 & $A$ & SLC16A9 & 0.080 & 0.014 & $1.00 \times 10^{-8}$ & 3,498 & 11,009 & 0.010 & 0.029 & 0.657 \\
\hline rs17300741 & A & SLC22A11 & 0.060 & 0.008 & $7.00 \times 10^{-14}$ & 3,498 & 11,009 & -0.020 & 0.022 & 0.259 \\
\hline rs2231142 & $\mathrm{T}$ & ABCG2 & 0.140 & 0.022 & $1.00 \times 10^{-10}$ & 3,498 & 11,009 & -0.094 & 0.034 & 0.006 \\
\hline rs734553 & $\mathrm{T}$ & SLC2A9 & 0.400 & 0.013 & $1.00 \times 10^{-192}$ & 3,498 & 11,009 & 0.010 & 0.026 & 0.679 \\
\hline rs742132 & A & SCGN & 0.050 & 0.009 & $9.00 \times 10^{-9}$ & 3,498 & 11,009 & 0.030 & 0.021 & 0.169 \\
\hline rs780094 & $\mathrm{T}$ & GCKR & 0.050 & 0.008 & $1.00 \times 10^{-9}$ & 3,498 & 11,009 & -0.030 & 0.020 & 0.190 \\
\hline \multicolumn{11}{|l|}{ B. Gout } \\
\hline \multirow{2}{*}{$\begin{array}{l}\text { Instrumental } \\
\text { SNP }\end{array}$} & \multirow{2}{*}{$\begin{array}{l}\text { Effect } \\
\text { allele }\end{array}$} & \multirow{2}{*}{ Gene } & \multicolumn{3}{|c|}{ Exposure (gout) } & \multicolumn{5}{|c|}{ Outcome (osteoarthritis) } \\
\hline & & & Beta & SE & p-value & Case (n) & Control (n) & Beta & SE & $p$-value \\
\hline rs1014290 & $\mathrm{T}$ & SLC2A9 & 0.451 & 0.042 & $7.00 \times 10^{-26}$ & 3,498 & 11,009 & 0.041 & 0.024 & 0.075 \\
\hline rs10791821 & G & MAP3K11 & 0.451 & 0.084 & $1.00 \times 10^{-7}$ & 3,498 & 11,009 & 0.000 & 0.026 & 0.973 \\
\hline rs1165176 & G & SLC17A1 & 0.351 & 0.057 & $1.00 \times 10^{-9}$ & 3,498 & 11,009 & 0.010 & 0.021 & 0.717 \\
\hline rs11733284 & A & NIPAL1 & 0.215 & 0.045 & $9.00 \times 10^{-7}$ & 3,498 & 11,009 & -0.041 & 0.024 & 0.099 \\
\hline rs11758351 & G & HIST1H2BF & 0.336 & 0.058 & $2.00 \times 10^{-8}$ & 3,498 & 11,009 & 0.049 & 0.031 & 0.088 \\
\hline rs1260326 & $\mathrm{T}$ & GCKR & 0.307 & 0.043 & $2.00 \times 10^{-12}$ & 3,498 & 11,009 & -0.041 & 0.024 & 0.089 \\
\hline rs2231142 & $\mathrm{T}$ & ABCG2 & 0.513 & 0.075 & $3.00 \times 10^{-12}$ & 3,498 & 11,009 & -0.094 & 0.034 & 0.006 \\
\hline rs2728125 & $\mathrm{C}$ & ABCG2 & 0.713 & 0.046 & $7.00 \times 10^{-54}$ & 3,498 & 11,009 & -0.073 & 0.036 & 0.043 \\
\hline rs3114020 & $\mathrm{C}$ & ABCG2 & 0.637 & 0.051 & $9.00 \times 10^{-35}$ & 3,498 & 11,009 & -0.030 & 0.021 & 0.193 \\
\hline rs4073582 & G & CNIH-2 & 0.507 & 0.086 & $6.00 \times 10^{-9}$ & 3,498 & 11,009 & 0.020 & 0.024 & 0.259 \\
\hline rs4766566 & $\mathrm{T}$ & CUX2 & 0.412 & 0.046 & $4.00 \times 10^{-20}$ & 3,498 & 11,009 & 0.030 & 0.026 & 0.201 \\
\hline rs734553 & $\mathrm{T}$ & SLC2A9 & 0.329 & 0.065 & $2.00 \times 10^{-7}$ & 3,498 & 11,009 & 0.010 & 0.026 & 0.652 \\
\hline
\end{tabular}

SNP: single nucleotide polymorphism, GWAS: genome-wide association study, Beta: beta coefficient, SE: standard error, SLC16A9: Solute Carrier Family 16 Member 9, SLC22A11: Solute Carrier Family 22 Member 11, ABCG2: ATP Binding Cassette Subfamily G Member 2 (Junior Blood Group), SLC2A9: Solute Carrier Family 2 Member 9, SCGN: Secretagogin, EF-Hand Calcium Binding Protein, GCKR: Glucokinase Regulator, MAP3K11: Mitogen-Activated Protein Kinase Kinase Kinase 11, SLC17A1: Solute Carrier Family 17 Member 1, NIPAL1: NIPA-Like Domain Containing 1, HIST1H2BF: Histone Cluster 1 H2B Family Member F, CNIH-2: Cornichon Family AMPA Receptor Auxiliary Protein 2, CUX2: Cut-Like Homeobox 2. 
A

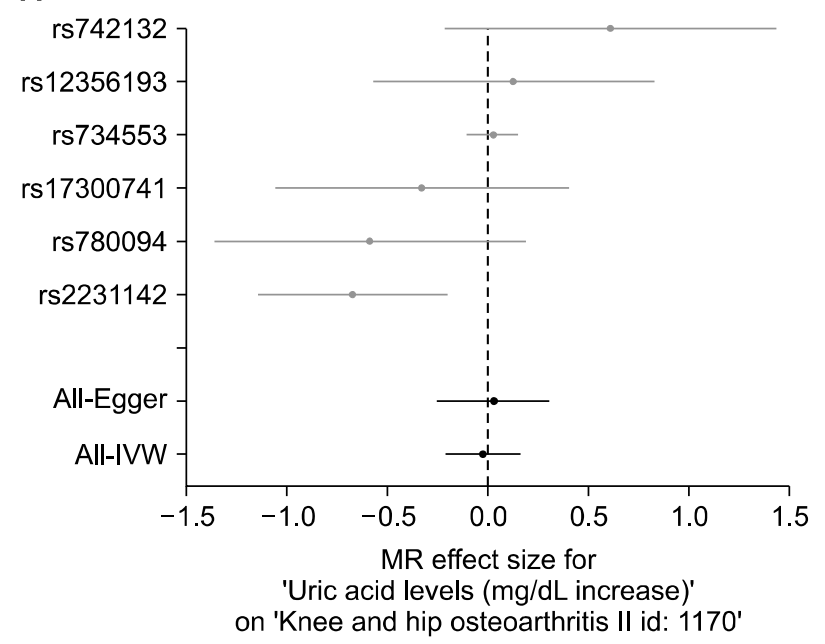

B

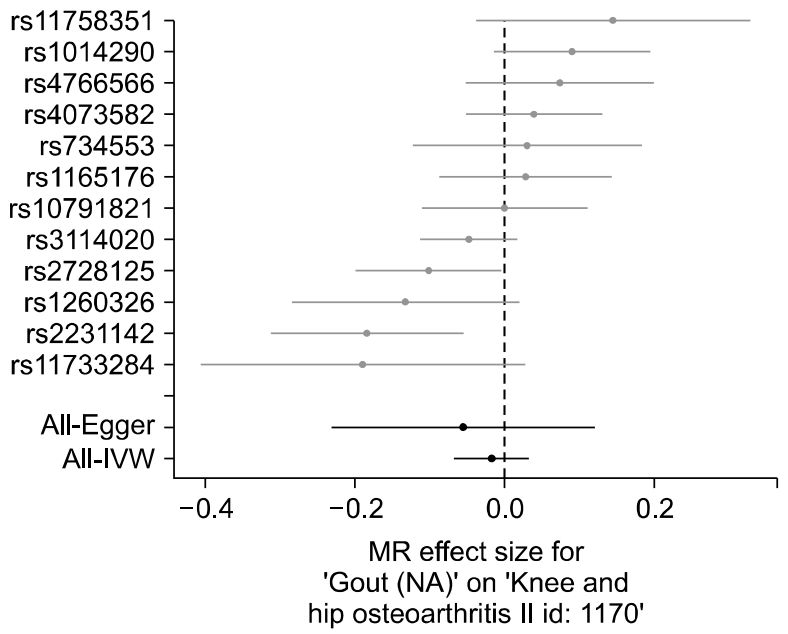

Figure 1. Forrest plot of the causal effects of uric acid level (A) or gout (B)-associated SNPs on osteoarthritis. SNP: single nucleotide polymorphism, MR: Mendelian randomization, IVW: inverse-variance weighted, Na: not available.

Table 2. The MR estimates of the causal effect of uric acid level (A) and gout (B) on osteoarthritis risk, derived using different methods

\begin{tabular}{|c|c|c|c|c|c|c|}
\hline \multicolumn{7}{|l|}{ A. Uric acid level } \\
\hline MR method & $\begin{array}{c}\text { Number of } \\
\text { SNP }\end{array}$ & Beta & SE & $\begin{array}{c}\text { Association } \\
\text { p-value }\end{array}$ & $\begin{array}{c}\text { Cochran Q } \\
\text { statistic }\end{array}$ & $\begin{array}{c}\text { Heterogeneity } \\
\text { p-value }\end{array}$ \\
\hline Inverse variance weighted & 6 & -0.026 & 0.096 & 0.789 & 13.02 & 0.232 \\
\hline MR-Egger & 6 & 0.028 & 0.142 & 0.852 & 12.09 & 0.166 \\
\hline Weighted median & 6 & 0.004 & 0.064 & 0.946 & na & na \\
\hline \multicolumn{7}{|l|}{ B. Gout } \\
\hline MR method & $\begin{array}{c}\text { Number of } \\
\text { SNP }\end{array}$ & Beta & SE & $\begin{array}{c}\text { Association } \\
\text { p-value }\end{array}$ & $\begin{array}{c}\text { Cochran Q } \\
\text { statistic }\end{array}$ & $\begin{array}{c}\text { Heterogeneity } \\
\text { p-value }\end{array}$ \\
\hline Inverse variance weighted & 12 & -0.018 & 0.025 & 0.482 & 26.53 & 0.005 \\
\hline MR-Egger & 12 & -0.056 & 0.090 & 0.548 & 26.03 & 0.004 \\
\hline Weighted median & 12 & -0.005 & 0.025 & 0.834 & na & na \\
\hline
\end{tabular}

MR: Mendelian randomization, SNP: single nucleotide polymorphism, Beta: beta coefficient, SE: standard error, na: not available.

\section{2) Mendelian randomization results}

The IVW method yielded no evidence to support a causal association between uric acid level or gout and the risk of osteoarthritis (beta $=-0.026, \mathrm{SE}=0.096, \mathrm{p}=0.789$; beta $=-0.018, S E=0.025, p=0.482$ ) (Table 2, Figures 1, 2 ). In the MR-Egger test, the intercept represents the average pleiotropic effect across the genetic variants, i.e., the average direct effect of a variant on the outcome. If the intercept differs from zero, it serves as an evidence of directional pleiotropy. MR-Egger regression revealed that directional pleiotropy was unlikely to be biasing uric acid level (intercept $=-0.013, p=0.610$ ) or uric acid results (intercept $=0.018, p=0.668$ ), while yielding no causal as- sociation between uric acid level or gout and the risk of osteoarthritis (beta $=0.028, \mathrm{SE}=0.142, \mathrm{p}=0.852$; beta $=$ $-0.056, \mathrm{SE}=0.090, \mathrm{p}=0.548$ ) (Table 2, Figures 1, 2). In addition, the weighted median approach provided no evidence of a causal association between uric acid level or gout and the risk of osteoarthritis (beta $=0.004, \mathrm{SE}=$ 0.064, $\mathrm{p}=0.946$; beta $=-0.005, \mathrm{SE}=0.025, \mathrm{p}=0.843$ ) (Table 2, Figures 1, 2). We performed subgroup analysis using exposure dataset of European. However, the MR result from European did not change significantly the overall MR result. 

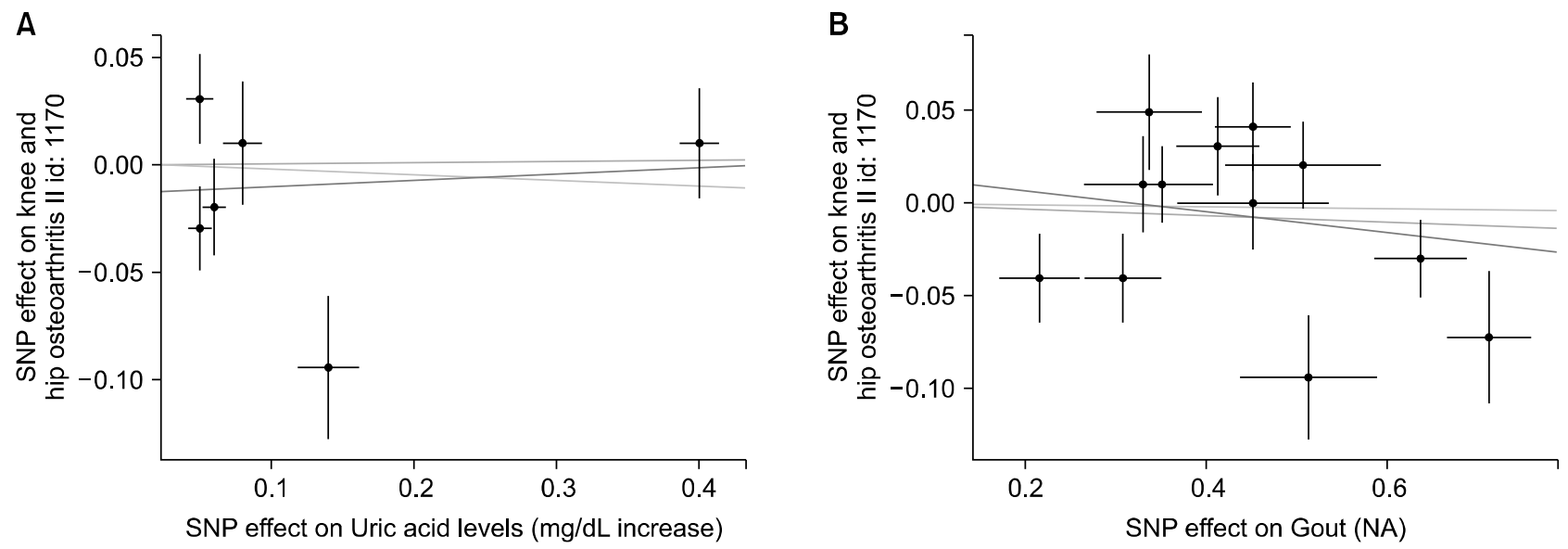

Figure 2. Scatter plots of genetic associations of uric acid level (A) or gout (B) against the genetic associations of osteoarthritis. The slopes of each line represent the causal association for each method. Blue line represents the IVW estimate, green line represents the weighted median estimate, and dark blue line represents the MR-Egger estimate. IVW: inverse-variance weighted, SNP: single nucleotide polymorphism, MR: Mendelian randomization, Na: not available.
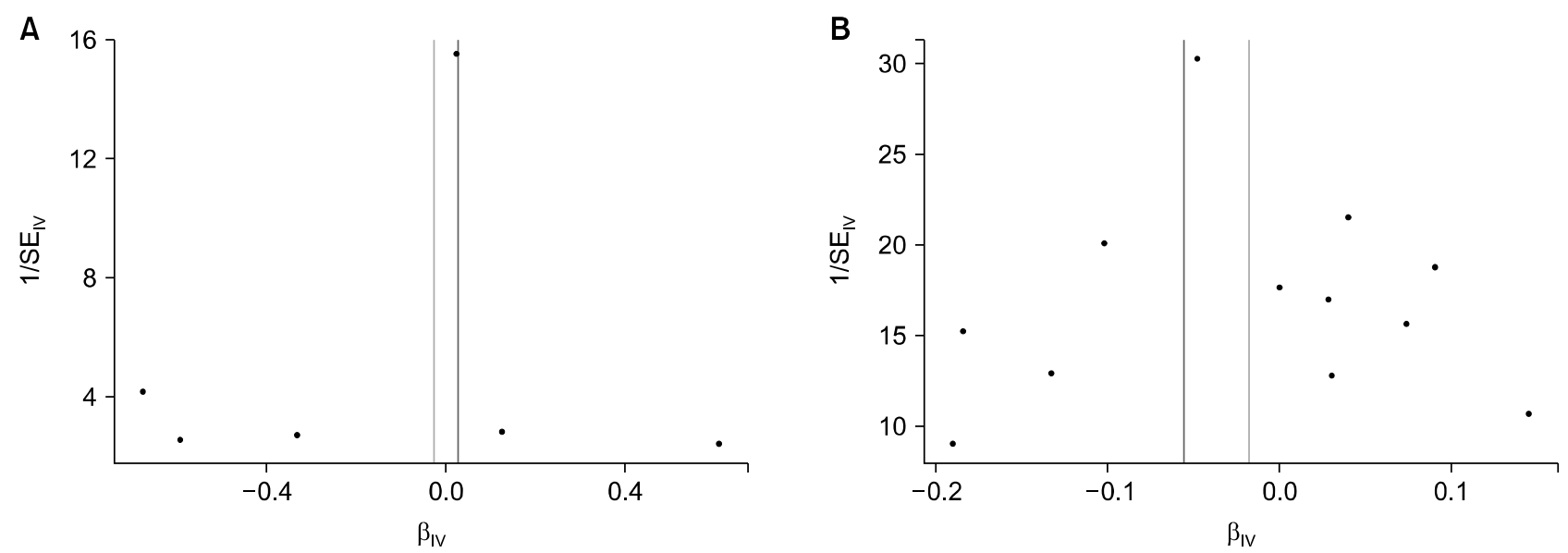

Figure 3. Funnel plot to assess the heterogeneity of the causal effects of uric acid level (A) or gout (B)-associated SNPs on osteoarthritis. Blue line represents the IVW estimate, and dark blue line represents the MR-Egger estimate. SNP: single nucleotide polymorphism, IVW: inverse-variance weighted, MR: Mendelian randomization, SE: standard error, $\beta$ : beta coefficient.

\section{3) Heterogeneity and sensitivity test}

Cochran's Q-test indicated no evidence of heterogeneity between IV estimates based on the individual variants of uric acid level, but not gout (Table 2). The funnel test yielded symmetry, indicating no evidence of heterogeneity in the MR analyses of uric acid level and gout (Figure 3). Results from the "leave one out" analysis demonstrated that no single SNP was driving the IVW point estimate.

\section{DISCUSSION}

Hyperuricemia and gout have been hypothesized to be associated with the development of osteoarthritis [23].
However, it remains unclear whether hyperuricemia and gout have a causal relationship with osteoarthritis, particularly because the previously reported associations between hyperuricemia or gout and osteoarthritis may be affected by the bias or confounding factors inherent to observational studies, reverse causation, limited number of studies with small sample sizes, and selection bias $[24,25]$. To investigate this, we employed three different estimation methods (inverse variance weighting method, weighted median method, and MR-Egger regression) for the MR analyses. The results of these methods were consistent with each another, yielding no causal associations between uric acid level or gout and osteoarthritis.

MR eliminates, or at least minimizes, the possibility of 
the bias inherent to observational studies due to residual confounding or reverse causality [26]. The assumption in $\mathrm{MR}$ is that the genetic variant associated with exposure is not associated with confounding variants and that the genetic variant influences the outcome only through the risk factor, thus, implying a causal effect of exposure on the outcome [27]. MR is a powerful tool for dissecting causal relationships between exposures and outcomes, owing to its minimization of residual confounding. However, MR studies are susceptible to bias resulting from pleiotropy [28]. Genetic variants may be associated with multiple phenotypes, a phenomenon known as 'pleiotropy', which may cause a confounded estimate from MR, potentially leading to biased causal estimates [29]. Although the inclusion of multiple variants in MR analysis typically leads to increased statistical power, it can also potentially lead to the inclusion of pleiotropic genetic variants that are not valid IVs [30]. Therefore, sensitivity analysis is needed to test the validity of conclusions from MR analysis. To eliminate pleiotropy, we employed a weighted median estimator, which provides valid estimates even if $50 \%$ of the SNPs are not valid instruments [20], and MR-Egger regression, which tests for unbalanced pleiotropy and estimates the causal effect of an exposure on an outcome [18]. Based on the intercept P-values obtained in this study, the MR-Egger approach showed no evidence of unbalanced pleiotropy. Use of the MR-Egger method may result in loss of precision and power, but the results of this analysis are supported by the results of the weighted median estimator analysis and similar to the IVW estimates, thus providing additional confidence in these findings. Genetic variants in MR are treated similarly to studies in a meta-analysis. Each genetic variant contributes an estimate of the causal effect and a pooled estimate is calculated based on all the genetic variants using a formula from the meta-analysis in MR. Thus, pooled estimate from MR is considered more accurate than estimate from each genetic variant by increasing the number of instruments, although the finding that the individual SNPs in ABCG2 are associated with OA are not negligible.

Although observational studies have provided evidence of association between uric acid level or gout and osteoarthritis [5,6], the results of our MR analysis suggest otherwise. Instead, the findings of the present study suggest that previous observational epidemiological associations may have been the result of confounding or reverse causation. In addition, our findings indicate the need for investigations into discrepancies between observational evidence and the absence of causal evidence. Such discrepancies could be accounted for by residual confounds in observational studies, which are unable to fully control for it.

The present study has several limitations. First, our analysis utilized a relatively small number of SNPs as IVs and may have limited power for detecting associations. The six SNPs associated with uric acid level explain only a relatively small proportion of the variation in vitamin $\mathrm{D}$ concentration [31]. This limits the statistical power for detection of a weak association between genetically predicted uric acid levels and osteoarthritis. The statistical power can be increased and a more precise causal estimate can be obtained by combining multiple genetic variants [32]. Second, the osteoarthritis data were from participants of European ancestry. As causality may depend on ethnicity, further MR studies based on other populations are needed. Third, only the osteoarthritis of knee and hip were included in this MR analysis. Osteoarthritis in other sites needs to be further explored. Fourth, since prevalence of gout and osteoarthritis are quite different according to sex, consideration of sex is needed. Unfortunately, we could not conduct the MR analysis based on sex, because the data from exposure and outcome did not provide the according to gender. Nevertheless, this meta-analysis has some strengths. First, although the uric acid level has been studied as a potential biomarker for osteoarthritis, to our knowledge, an MR analysis has never been performed. Therefore, this is the first MR study investigating the potential role of uric acid and gout in the development of osteoarthritis, yielding no association between genetically predicted uric acid level or gout and osteoarthritis. Second, using a large genetic data of osteoarthritis risk (consisting of 3,498 patients with osteoarthritis of the knee and hip and 11,009 controls) enabled us to test our study hypothesis more precisely, which would have not been possible with individual-level data from a smaller study.

\section{CONCLUSION}

In conclusion, the results of our MR analysis demonstrates that uric acid level and gout may be not causally associated with the increased risk of osteoarthritis. Considering MR study is not susceptible to bias from unmeasured confounders or reverse causation, the epidemiological evidence for an association between uric 
acid level or gout and a higher risk of osteoarthritis may be due to residual confounding or reverse causation rather than direct causality. Well-designed epidemiological and MR studies using more variants that explain a greater proportion of uric acid level or gout are warranted to confirm or rule out its causal relationship with osteoarthritis.

\section{CONFLICT OF INTEREST}

No potential conflict of interest relevant to this article was reported..

\section{AUTHOR CONTRIBUTIONS}

Y.H.L. was involved in conception and design of study, acquisition of data, analysis and interpretation of data, drafting the manuscript, and revising the manuscript. G.G.S. was involved in conception and design of study, analysis and interpretation of data, and drafting the manuscript.

\section{REFERENCES}

1. Dieppe PA, Lohmander LS. Pathogenesis and management of pain in osteoarthritis. Lancet 2005;365:965-73.

2. Litwic A, Edwards MH, Dennison EM, Cooper C. Epidemiology and burden of osteoarthritis. Br Med Bull 2013;105:185-99.

3. Wortmann RL. Gout and hyperuricemia. Curr Opin Rheumatol 2002;14:281-6.

4. Terkeltaub RA. Clinical practice. Gout. N Engl J Med 2003;349:1647-55.

5. Ding X, Zeng C, Wei J, Li H, Yang T, Zhang Y, et al. The associations of serum uric acid level and hyperuricemia with knee osteoarthritis. Rheumatol Int 2016;36:567-73.

6. Howard RG, Samuels J, Gyftopoulos S, Krasnokutsky S, Leung J, Swearingen CJ, et al. Presence of gout is associated with increased prevalence and severity of knee osteoarthritis among older men: results of a pilot study. J Clin Rheumatol 2015;21:63-71.

7. Dalbeth N, Aati O, Kalluru R, Gamble GD, Horne A, Doyle AJ, et al. Relationship between structural joint damage and urate deposition in gout: a plain radiography and dual-energy CT study. Ann Rheum Dis 2015;74:1030-6.

8. Yokose C, Chen M, Berhanu A, Pillinger MH, Krasnokutsky S. Gout and osteoarthritis: associations, pathophysiology, and therapeutic implications. Curr Rheumatol Rep 2016; 18:65.

9. Burgess S, Daniel RM, Butterworth AS, Thompson SG; EPIC-InterAct Consortium. Network Mendelian randomization: using genetic variants as instrumental variables to investigate mediation in causal pathways. Int J Epidemiol 2015;44:484-95.

10. Kolz M, Johnson T, Sanna S, Teumer A, Vitart V, Perola M, et al. Meta-analysis of 28,141 individuals identifies common variants within five new loci that influence uric acid concentrations. PLoS Genet 2009;5:e1000504.

11. Sulem P, Gudbjartsson DF, Walters GB, Helgadottir HT, Helgason A, Gudjonsson SA, et al. Identification of low-frequency variants associated with gout and serum uric acid levels. Nat Genet 2011;43:1127-30.

12. Matsuo H, Yamamoto K, Nakaoka H, Nakayama A, Sakiyama M, Chiba T, et al. Genome-wide association study of clinically defined gout identifies multiple risk loci and its association with clinical subtypes. Ann Rheum Dis 2016;75:652-9.

13. Nakayama A, Nakaoka H, Yamamoto K, Sakiyama M, Shaukat A, Toyoda Y, et al. GWAS of clinically defined gout and subtypes identifies multiple susceptibility loci that include urate transporter genes. Ann Rheum Dis 2017;76: 869-77.

14. arcOGEN Consortium; arcOGEN Collaborators, Zeggini E, Panoutsopoulou K, Southam L, Rayner NW, Day-Williams AG, Lopes MC, et al. Identification of new susceptibility loci for osteoarthritis (arcOGEN): a genome-wide association study. Lancet 2012;380:815-23.

15. Burgess S, Butterworth A, Thompson SG. Mendelian randomization analysis with multiple genetic variants using summarized data. Genet Epidemiol 2013;37:658-65.

16. Hartwig FP, Davies NM, Hemani G, Davey Smith G. Two-sample Mendelian randomization: avoiding the downsides of a powerful, widely applicable but potentially fallible technique. Int J Epidemiol 2016;45:1717-26.

17. Pierce BL, Burgess S. Efficient design for Mendelian randomization studies: subsample and 2-sample instrumental variable estimators. Am J Epidemiol 2013;178:1177-84.

18. Bowden J, Davey Smith G, Burgess S. Mendelian randomization with invalid instruments: effect estimation and bias detection through Egger regression. Int $\mathrm{J}$ Epidemiol 2015;44:512-25.

19. Burgess S, Thompson SG. Interpreting findings from Mendelian randomization using the MR-Egger method. Eur J Epidemiol 2017;32:377-89.

20. Bowden J, Davey Smith G, Haycock PC, Burgess S. Consistent estimation in Mendelian randomization with some invalid instruments using a weighted median estimator. Genet Epidemiol 2016;40:304-14.

21. Hemani G, Zheng J, Wade KH, Laurin C, Elsworth B, Burgess S, et al. MR-Base: a platform for systematic causal inference across the phenome using billions of genetic associations. bioRxiv 2016;16:78972.

22. Egger M, Smith GD, Phillips AN. Meta-analysis: principles and procedures. BMJ 1997;315:1533-7.

23. Neogi T, Krasnokutsky S, Pillinger MH. Urate and osteoarthritis: Evidence for a reciprocal relationship. Joint Bone Spine 2019;86:576-82.

24. Hill HA, Kleinbaum DG. Bias in observational studies. Wiley StatsRef 2000. DOI: 10.1002/9781118445112. stat05111.

25. Lee YH, Bae SC, Song GG. Hepatitis B virus (HBV) reactivation in rheumatic patients with hepatitis core antigen (HBV occult carriers) undergoing anti-tumor necrosis factor therapy. Clin Exp Rheumatol 2013;31:118-21.

26. Smith GD, Ebrahim S. Mendelian randomization: genetic variants as instruments for strengthening causal inference in observational studies. In: Weinstein M, Vaupel JW, 
Wachter KW, eds. Biosocial surveys. Washington D.C., National Academies Press (US), 2008.

27. VanderWeele TJ, Tchetgen Tchetgen EJ, Cornelis M, Kraft P. Methodological challenges in Mendelian randomization. Epidemiology 2014;25:427-35.

28. Thompson JR, Minelli C, Bowden J, Del Greco FM, Gill D, Jones EM, et al. Mendelian randomization incorporating uncertainty about pleiotropy. Stat Med 2017;36:4627-45.

29. Paaby AB, Rockman MV. The many faces of pleiotropy. Trends Genet 2013;29:66-73.

30. Smith GD, Ebrahim S. Mendelian randomization: pros- pects, potentials, and limitations. Int J Epidemiol 2004;33: $30-42$.

31. Swerdlow DI, Kuchenbaecker KB, Shah S, Sofat R, Holmes MV, White J, et al. Selecting instruments for Mendelian randomization in the wake of genome-wide association studies. Int J Epidemiol 2016;45:1600-16.

32. Burgess S, Dudbridge F, Thompson SG. Combining information on multiple instrumental variables in Mendelian randomization: comparison of allele score and summarized data methods. Stat Med 2016;35:1880-906. 